\title{
Finite element simulation of the manufacturing process chain of a sheet metal assembly
}

\author{
Alexander Govik, Larsgunnar Nilsson and Ramin Moshfegh
}

\section{Linköping University Post Print}

N.B.: When citing this work, cite the original article.

Original Publication:

Alexander Govik, Larsgunnar Nilsson and Ramin Moshfegh, Finite element simulation of the manufacturing process chain of a sheet metal assembly, 2012, Journal of Materials Processing Technology, (212), 7, 1453-1462.

http://dx.doi.org/10.1016/j.jmatprotec.2012.02.012

Copyright: Elsevier

http://www.elsevier.com/

Postprint available at: Linköping University Electronic Press

http://urn.kb.se/resolve?urn=urn:nbn:se:liu:diva-77853 


\title{
Finite element simulation of the manufacturing process chain of a sheet metal assembly
}

\author{
Alexander Govik ${ }^{\mathrm{a}, *}$, Larsgunnar Nilsson ${ }^{\mathrm{a}}$, Ramin Moshfegh ${ }^{\mathrm{a}, \mathrm{b}}$ \\ ${ }^{a}$ Division of Solid Mechanics, Linköping University, SE-58183 Linköping, Sweden \\ ${ }^{b}$ Outokumpu Stainless AB, Avesta, Sweden
}

\begin{abstract}
An increasing number of components in automotive structures are today made from advanced high strength steel (AHSS). Since AHSS demonstrates more severe springback behaviour than ordinary mild steels, it requires more efforts to meet the design specification of the stamped parts. Consequently, the physical fine tuning of the die design and the stamping process can be time consuming. The trial-and-error development process may be shortened by replacing most of the physical try-outs with finite e lement (FE) simulations of the forming process, including the springback behaviour. Still it can be hard to identify when a stamped part will lead to an acceptable assembly with respect to the geometry and the residual stress state. In part since the assembling process itself will distort the components. To resolve this matter it is here proposed to extend the FE-simulation of the stamping process, to also include the first level sub-assembly stage. In this study a methodology of sequentially simulating each step in the manufacturing process of an assembly is proposed. Each step of the proposed methodology is described, and a validation of the prediction capabilities is performed by comparing with a physically manufactured assembly. The assembly is composed of three sheet metal components made from DP600 steel which are joined by spot welding. The components are designed to exhibit severe springback behaviour in order to put both the forming and subsequent assembling simulations to the test. The work presented here demonstrates that by using virtual prototyping it is possible to predict the final shape of an assembled structure.
\end{abstract}

Keywords:

Finite element simulation, Assembly, Sheet metal, Forming, Springback

\section{Introduction}

Increasing consumer demands for high safety, fuel-efficient and low emission vehicles, forces the automotive industry to increase its use of advanced high strength steel in various structural parts. This may cause problems during the manufacturing of such parts and their subsequent assembly since AHSS demonstrates more severe springback behaviour than ordinary mild steels. Consequently, much effort has to be put in to modifying the die and the stamping process in

${ }^{*}$ Corresponding author. Tel.:+46-13-281139; fax:+46-13-282717.

Email address: alexander .govik@liu.se (Alexander Govik) 
order to fulfil the design specification of each individual part. Virtual prototyping of the die try-out phase, based on FE simulations of the forming process, is an efficient methodology to manage these difficulties.

Already in the 1990's the automotive industry used FE simulations of sheet metal forming processes at the tool and process design stages, in order to predict forming defects such as wrinkles and tearing (see Makinouchi (1996) and Ahmetoglu et al. (1994)). The springback behaviour, however, could not be predicted with a satisfactory accuracy (Makinouchi et al. (1998)). Since then there has been an extensive focus on the accuracy of springback analysis. Both the effects of different FE modelling and numerical parameters as well as material modelling issues have been investigated. For example, Lee and Yang (1998) studied how the mesh density of the blank itself and at the tool draw-radii, as well as how the punch velocity influence the accuracy of the springback prediction. Papeleux and Ponthot (2002) investigated the effect on springback from e.g., blank holder force, the coefficient of friction, and the choice of time discretisation in the solution process. Li et al. (2002) studied how element formulation, the number of through-thickness integration points, and tool radius versus blank thickness affected the springback. Wagoner and $\mathrm{Li}$ (2007) studied the role of the number of through-thickness integration points by comparing the analytical and numerical integration of bending moments in the bending-under-tension of a beam.

In the field of material modelling a vast amount of research has been done. For detailed information, Banabic (2000) presented a comprehensive review of yield criteria, and Eggertsen and Mattiasson (2009) have investigated five different hardening laws and their influence on the springback prediction.

However, according to Hammett et al. (1998) the lack of a strong correlation between component dimension and first level sub-assembly dimension, suggests that reaching tight set tolerances for each component is not a guarantee that the sub-assemblies will reach their tolerances. Instead they proposed the use of the functional build method, where the stamped parts are not evaluated individually but rather collectively within their assembly. Thus, if the assembly meets its tolerances, an individual part can be approved even though it fails to reach its initial design specification. By implementing this method much die try-outs can evidently be avoided. However, many pre-production trials are still needed since each sub-assembly must be evaluated. A way to circumvent these physical try-outs is to replace them with virtual try-outs. Since the assembling process now can be seen as an integrated part of the die try-out stage, a natural step is to expand the virtual try-outs of the die and stamping process to also incorporate the first level sub-assembly stage.

In the field of geometry prediction of assemblies, the main work has been done in the analysis of dimension variations. These analyses are performed during early design stages to avoid variation-related production problems. They are based on measured physical parts, or in the early stages on an assumed deviation from the nominal geometry. Skowronski and Turner (1997) developed a statistical tolerance synthesis, though the deformation of the parts was not accounted for; Liu and $\mathrm{Hu}$ (1997) proposed a variation simulation method using a linear FEM, without considering contact between the parts; Cai et al. (2006) included contact analysis to avoid part penetration. Since all of these approaches require a large amount of simulations, the computation time is a concern. Thus there is still a significant amount of simplifications which will affect the accuracy of the results.

A deterministic FE simulation of the manufacturing process chain is another approach to evaluate the manufacturing process and the properties of the final assembly. Galbraith et al. (2003) studied the difference in springback of the individual parts compared to the springback 
of the whole assembly. In their study the assembling process was significantly simplified. After the forming simulation, but before springback, each sub-component was positioned into its assembled location and tied to the other components at the spot weld positions. A springback simulation was subsequently performed on the whole assembly. Even though the assembling process normally is elastic, the simplification in their approach entails that distortions caused by misalignment during the clamping process or by the welding process are neglected. $\mathrm{Pa}$ padakis (2008) studied the structural effects from welding of frame assemblies by taking the entire manufacturing process chain into account. The chaining of the FE simulations was realised by mapping the results from each previous step onto an FE mesh, based on the nominal geometry, prepared for the welding simulation. This approach entails that the deviation from the nominal geometry of each component must be small otherwise the mapping yields incorrect results. Zhang et al. (2009) presented modelling methods for the simulation of manufacturing processes of sheet metal assemblies. However no experimental validation of the simulations was presented.

Much information about the manufacturing process may be gained from FE simulations of the entire manufacturing process chain, i.e., from forming of the individual sub-components to the assembling process. Different fixture designs as well as clamping and welding sequences can be explored. Predictions can be made whether the final assembly will meet the design specification, or if some alteration of the assembling procedure or the stamping process of the individual components must be made.

In this contribution a methodology to predict the properties of a sheet metal assembly is presented. By sequentially simulate each step of the manufacturing process, the methodology can be applied to describe the evolution of deformations and residual stresses throughout the manufacturing process chain. The virtual prototyping process starts with the creation of FE models for the forming and trimming operations, including the associated springback, of the individual components. The FE components obtained are then positioned and clamped to the assembly fixture, and their flanges are clamped together. The flanges are joined with spot welds and, finally, a springback analysis of the complete assembled structure is carried out. The geometry of the assembled part obtained from the FE simulations is compared with the geometry of the part obtained from physical tests.

\section{Methodology}

During the manufacturing process of a sheet metal assembly each component is subjected to a number of operations that will alter its properties. The process can be divided into two distinct phases; first the forming of the individual sheet components and, secondly, the assembling of all components to make the complete assembled structure. These phases can in turn be further divided into sub-phases. The sheet forming process can include multiple stages of stamping, including trimming or other type of forming operations. The assembling process can typically be divided into four steps called the PCFR cycle: Place, Clamp, Fasten and Release. All these phases and sub-phases contribute to the transformation from blanks to an assembled structure. As a consequence, each step of the manufacturing process chain needs to be taken into account in order to predict the final properties of the assembled structure. An FE simulation procedure based on this conclusion has been developed (see Fig. 1).

A case study of the manufacturing of an assembly is presented in order to validate the prediction capabilities of the simulation procedure. In this study, an assembly consisting of three 


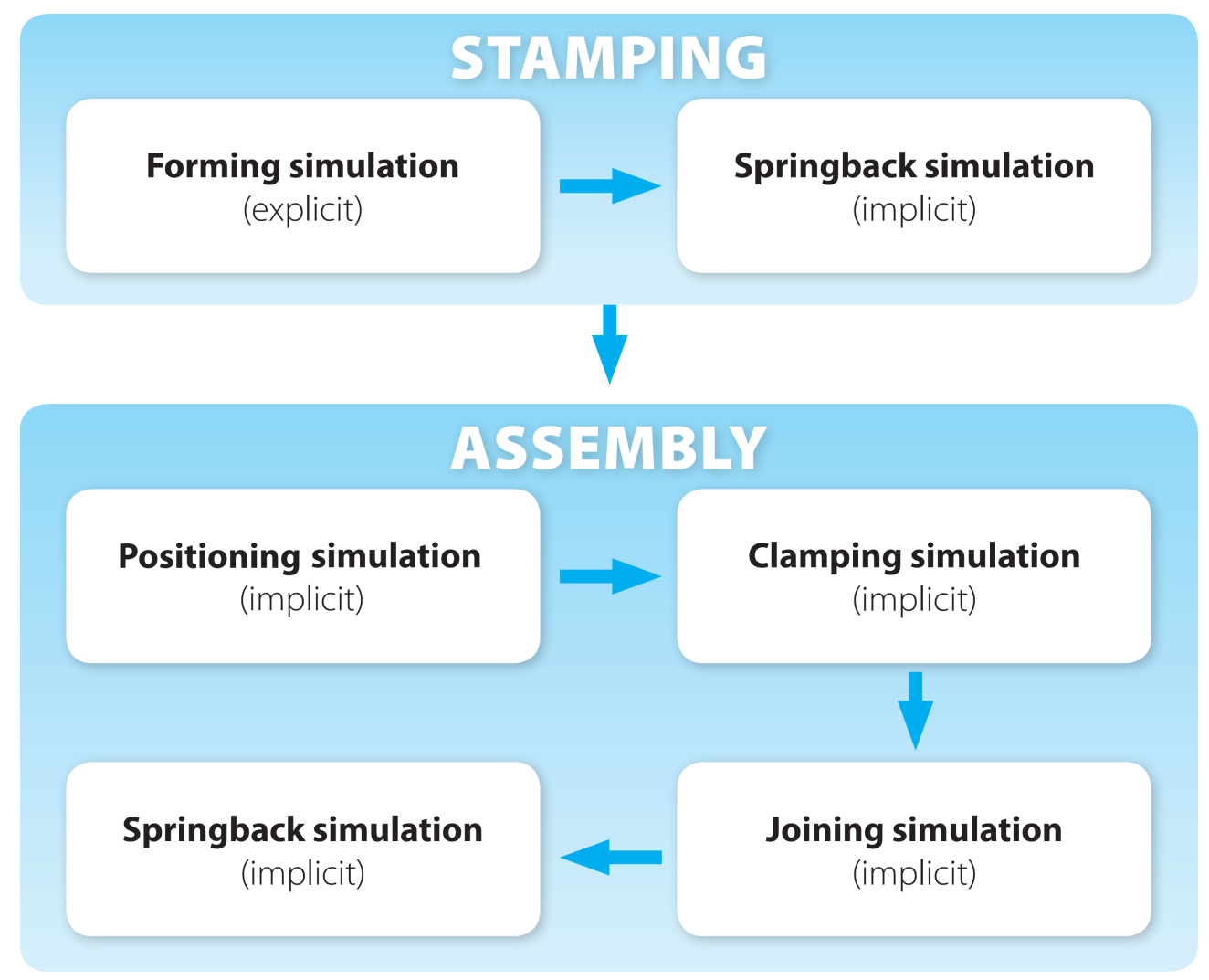

Figure 1: FE simulation procedure of the manufacturing process.

components according to Fig. 2 is used. The flex-rail tool (see Fig. 3) presented by Andersson (2007), is used for the stamping of all three components. The flex-rail tool was originally designed as a semi-industrial benchmark tool that produces a sheet component with a complex springback behaviour including flange/wall angle change, twist and sidewall curl. These failure modes are all characteristic problems of a complex automotive component and a reason why parts formed by this tool are chosen for this study. The components are then assembled according to the PCFR cycle.

\section{Experimental set-up}

The sheet components are made from $1.4 \mathrm{~mm}$ thick dual phase carbon steel DP600 using an initial blank size of $500 \mathrm{~mm}$ × $350 \mathrm{~mm}$, with the rolling direction along its shortest side. Component 1 is the net shape achieved by the stamping, while components 2 and 3 are the cut off ends from a stamped net shape component. 


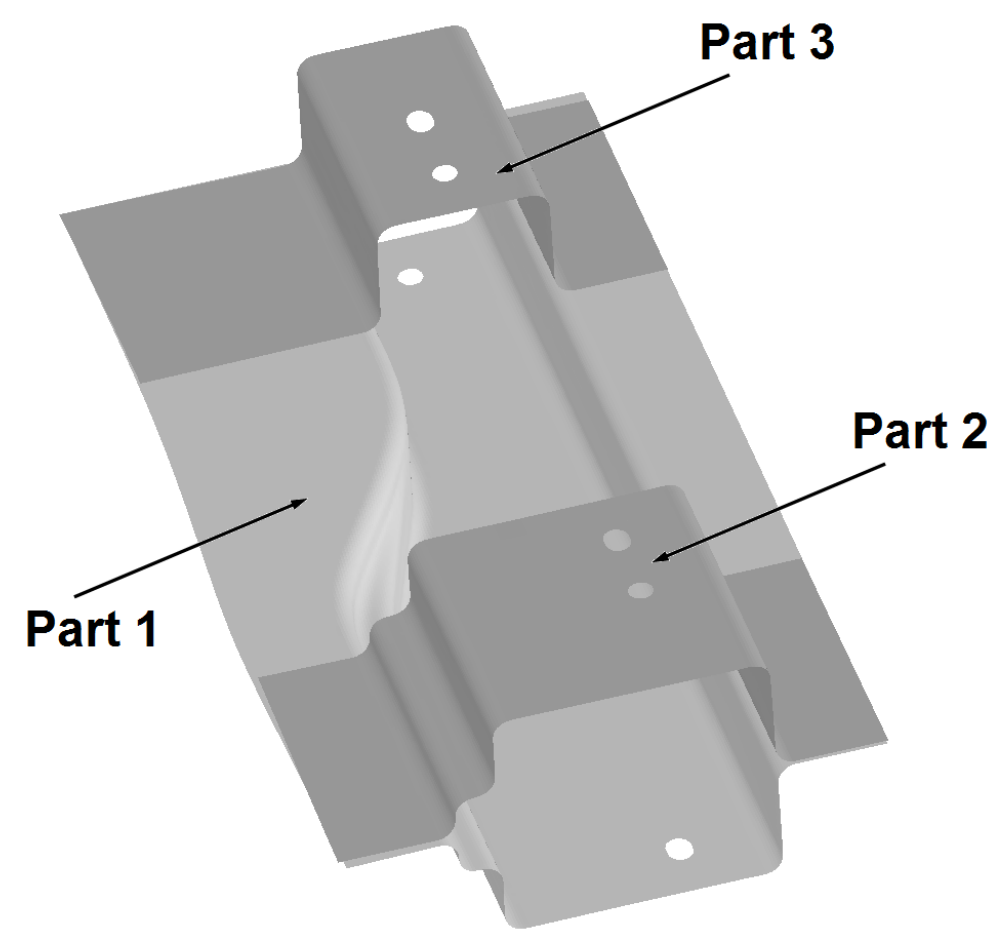

Figure 2: Assembly made from three parts.

\subsection{Stamping procedure}

The flex-rail tool is mounted in a hydraulic single action press and the draw depth is set to $60 \mathrm{~mm}$. The blank-holder force is $375 \mathrm{kN}$ at the beginning of the stroke and increases linearly to $510 \mathrm{kN}$ at the end. Lubricant is manually distributed over the blank surface. A marking device is incorporated in the flex-rail tool to achieve stable reference points. It marks positions on the blank in the bottom position of the punch motion. After the part is removed, a hole or a slot is made at the marked positions (see Fig. 2).

\subsection{Assembly procedure}

The assembling process is performed manually in accordance with the PCFR-cycle. The components are positioned in an assembly fixture based on the 3-2-1 locator scheme, i.e., each part is positioned by three vertical supports and the hole and slot are mated with corresponding reference pins. Then the components are clamped to the fixture at each vertical support by $\mathrm{C}$ clamp locking pliers, hence called clamps. Thereafter the flanges are clamped to each other with two clamps uniformly positioned on each flange of the components 2 and 3 according to Fig. 4. It should be noted that the components used in this semi-industrial case study demonstrate larger springback and clamping forces than normally would have been accepted in a real production 


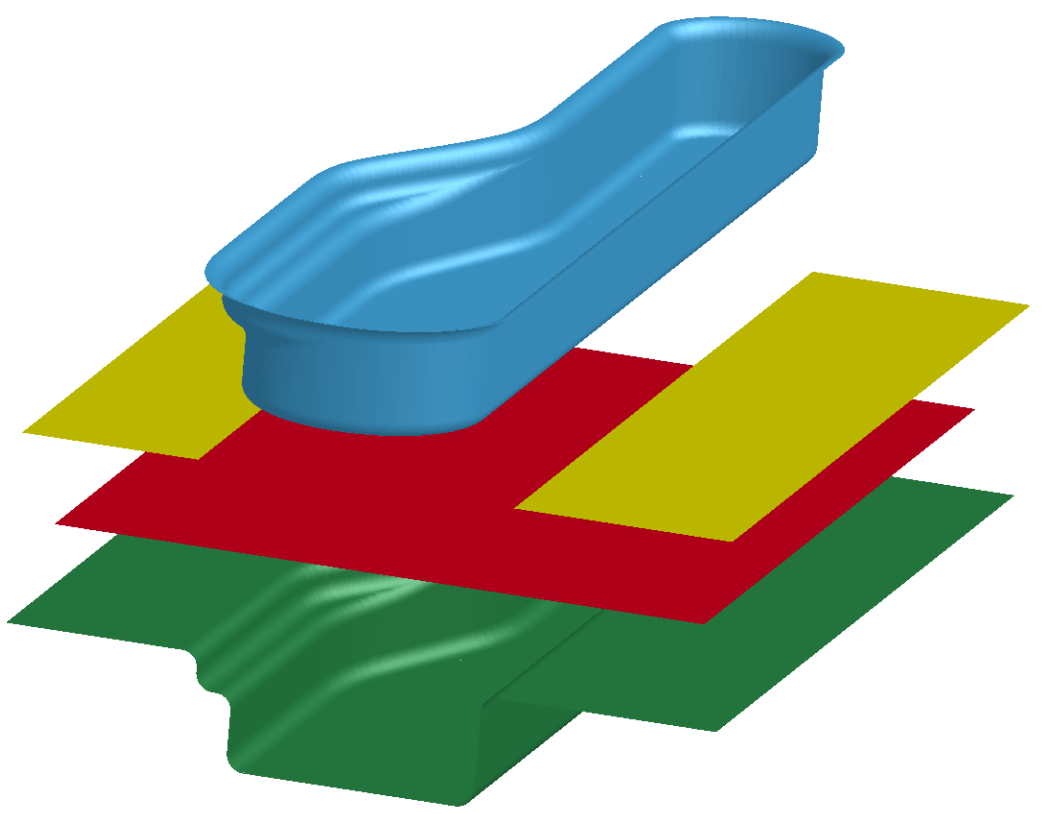

Figure 3: FE model of the flex-rail tool.

process. Three spot welds per flange are made using a handheld weld gun. Finally the clamps are released, first the clamps on the flanges then the clamps on the fixture.

\subsection{Measurement procedure}

Due to practical reasons the geometries of the individual components after stamping and trimming are measured while they are positioned and clamped in the assembly fixture. Hence, it is not the true springbacked geometries of the components that are measured; the clamping introduces a deformation that affects the twist of the components. The measurement is conducted using a coordinate measuring machine (CMM). Points along a number of lines in the width direction are measured to find the cross-section geometries (see Fig. 5).

The final assembled structure is measured using a different fixture (see Fig. 6). This fixture is also based on the 3-2-1 locator principle to achieve a stable reference system. Furthermore the assembly is not clamped to the fixture, i.e., the true unconstrained geometry of the assembly is measured.

\section{Material modelling}

During the typical stamping process the material behaviour is elasto-plastic, while the springback and the assembling processes usually are pure elastic processes. However, in order to retain all information from each previous process step, e.g., history variables of the material model, there is a need to be consistent in the material modelling. Thus, an elasto-plastic material model is used in the simulations of all process steps. 

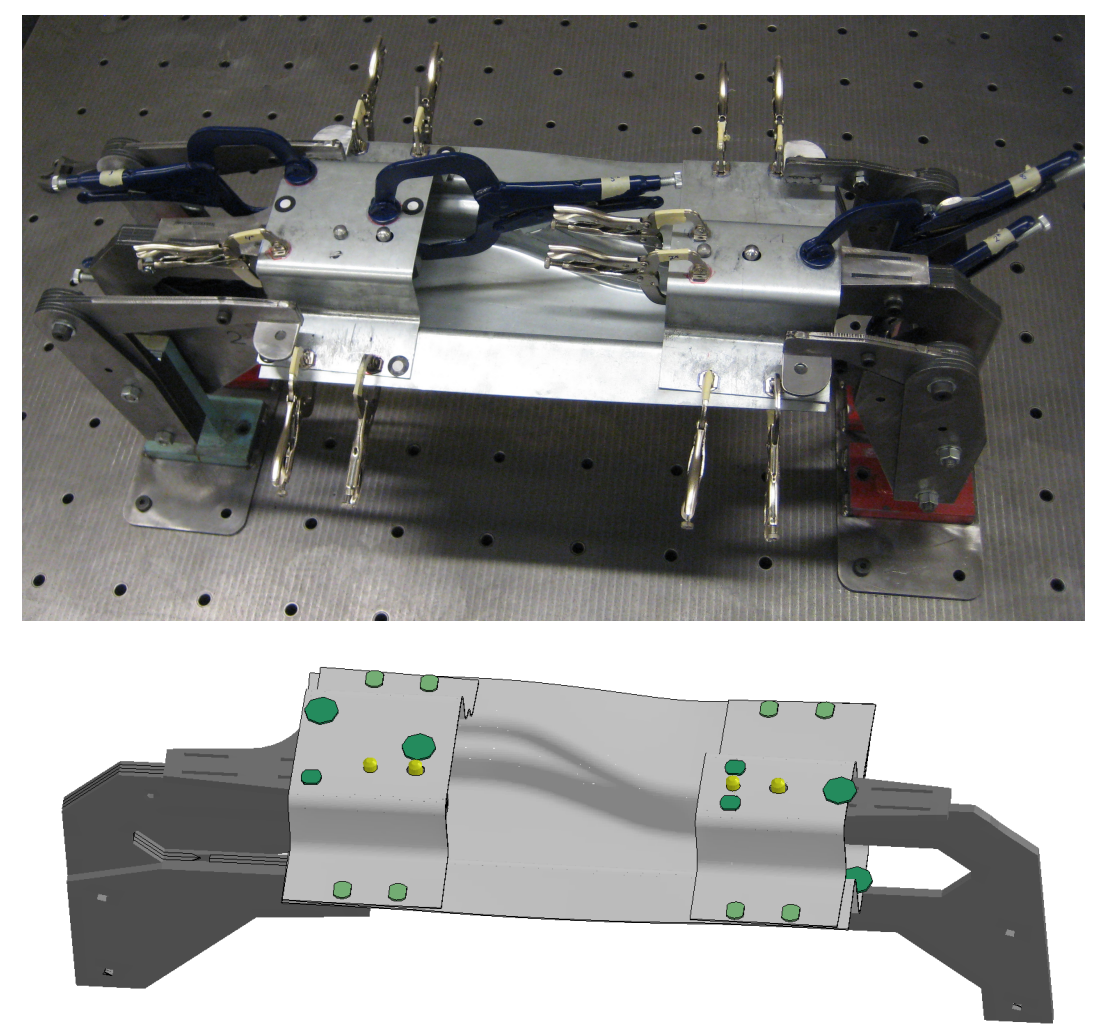

Figure 4: Components fully clamped in the assembly fixture, experimental and FE-model.

In order to achieve accurate results from simulations of the sheet forming process involving AHSS steel and its associated springback, advanced material models are mandatory. A number of subjects, such as yield condition and plastic hardening behaviour, are associated with the concept of material modelling.

As a result of the cold rolling manufacturing process of sheet metal, the material becomes plastically orthotropic, i.e., the inelastic properties are different along the rolling direction, the transversal direction, and the thickness direction. To account for this phenomenon a number of anisotropic yield criteria have been presented. The first of these was the Hill' 48 criterion (see Hill (1948)). Among various other yield criteria one could mention the widely used three parameter Yld89 criterion (see Barlat and Lian (1989)), and the more complex eight parameter Yld2000 criterion (see Barlat et al. (2003)).

During deep drawing the sheet material is subjected to a number of bending and unbending cycles depending on tool and drawbead layout. This may be the origin of several phenomena, e.g., Bauschinger effects and work-hardening stagnation (see Yoshida and Uemori (2003)). To achieve an accurate springback prediction the plastic hardening law of the material model must be able to handle most of these effects.

In a sheet metal forming process the plastic strain can reach high values, well beyond the strain at necking in a tensile test. There are several different approaches to extend the plastic 


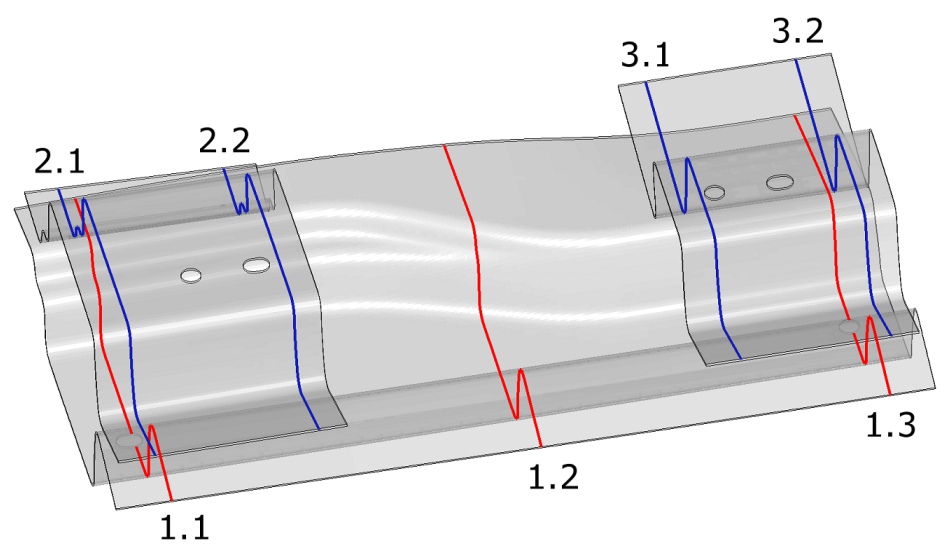

(a) Individual components after clamping to the fixture

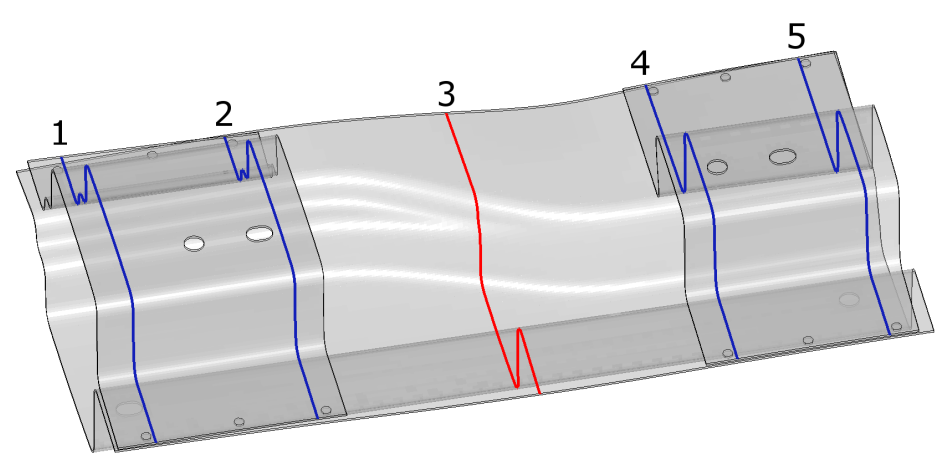

(b) Assembled structure

Figure 5: Cross-sections for CMM measuring.

hardening curve after necking. One approach is to extrapolate it by using one of the numerous mathematical expressions proposed in the literature. Another approach is to use experimental data from shear or biaxial testing, which typically can handle at least twice the strain range compared to a tensile test.

An often overlooked effect, in the simulation of springback of a stamped part, is the degradation of elastic stiffness due to the plastic deformation. The degradation can be substantial as shown by Eggertsen and Mattiasson (2010), who for a DP600 steel obtained a 25 percent decrease of the elastic stiffness at a level of 15 percent plastic strain. However, it has also been shown that this degradation eventually will recover. According to Morestin and Boivin (1996) the elastic stiffness will be recovered after 2-5 days depending on the type of steel. So in many cases this is an effect that only will affect the springback behaviour and not the forthcoming manufacturing steps. 


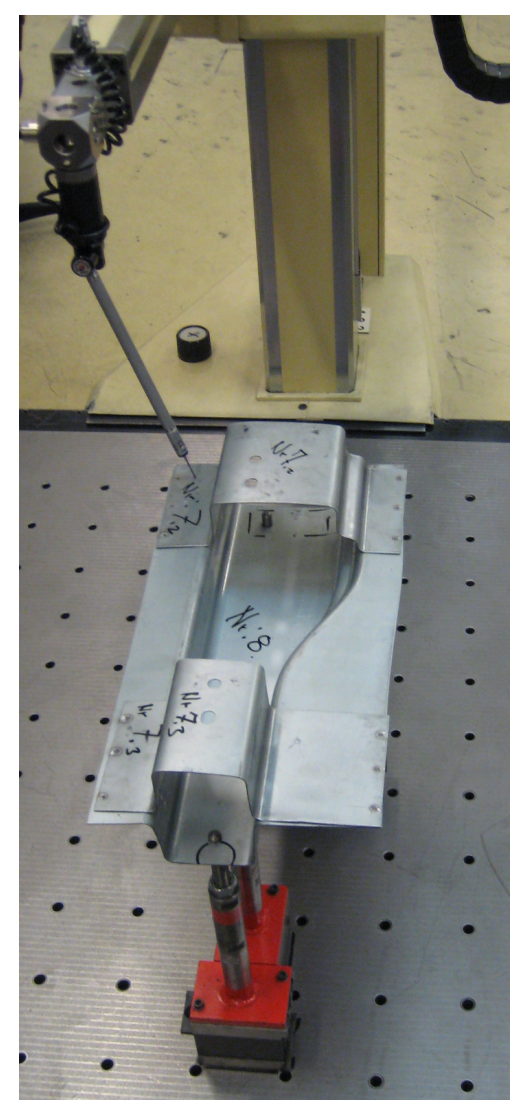

Figure 6: Measurement of the final assembly.

In this study a material model based on the Barlat YLD2000 yield criterion is used. The eight material parameters needed are obtained from uniaxial tensile tests in three different material directions, $\sigma_{0}, \sigma_{45}, \sigma_{90}, r_{0}, r_{45}, r_{90}$ and one bulge test, $\sigma_{b}, r_{b}$ (see Table 1 ). The stress component, $\sigma_{x x}$, and plastic strain ratio, $r_{x x}$, subscripts denote the specimen orientation relative to the rolling direction. The absence of drawbeads in the flex-rail tool will reduce the number of bending and unbending cycles to just one cycle. It is therefore reasonable to use a simple mixed isotropickinematic hardening, with a backstress evolution law of the Prager type, where a constant $\beta$ $[0,1]$ determines the fraction of kinematic hardening. The $\beta$ constant was given the value 0.26 , Eggertsen and Mattiasson (2009).

Experimental stress-strain curves are used to constitute the plastic hardening curve. A uniaxial tensile test curve is merged with an equibiaxial curve from a bulge test (see Fig. 7) by transforming the equibiaxial stress-strain data to effective ones. The transformation is achieved by scaling and fitting the equibiaxial data to the uniaxial data as described in Sigvant et al. (2009).

The elastic stiffness degradation is modelled in accordance with the model presented by Yoshida et al. (2002) with experimental values from Eggertsen and Mattiasson (2010). This degradation is only considered during the springback analysis; all the simulations of the assembling processes are performed using a constant Young's modulus found from the virgin steel 
Table 1: Material data.

\begin{tabular}{ccccccccc}
\hline$\sigma_{0}(\mathrm{MPa})$ & $\sigma_{45}(\mathrm{MPa})$ & $\sigma_{90}(\mathrm{MPa})$ & $\sigma_{b}(\mathrm{MPa})$ & $r_{0}$ & $r_{45}$ & $r_{90}$ & $r_{b}$ & Thickness $(\mathrm{mm})$ \\
\hline 386.1 & 399.7 & 414.8 & 405.8 & 0.76 & 0.93 & 0.95 & 0.99 & 1.4 \\
\hline
\end{tabular}

material.

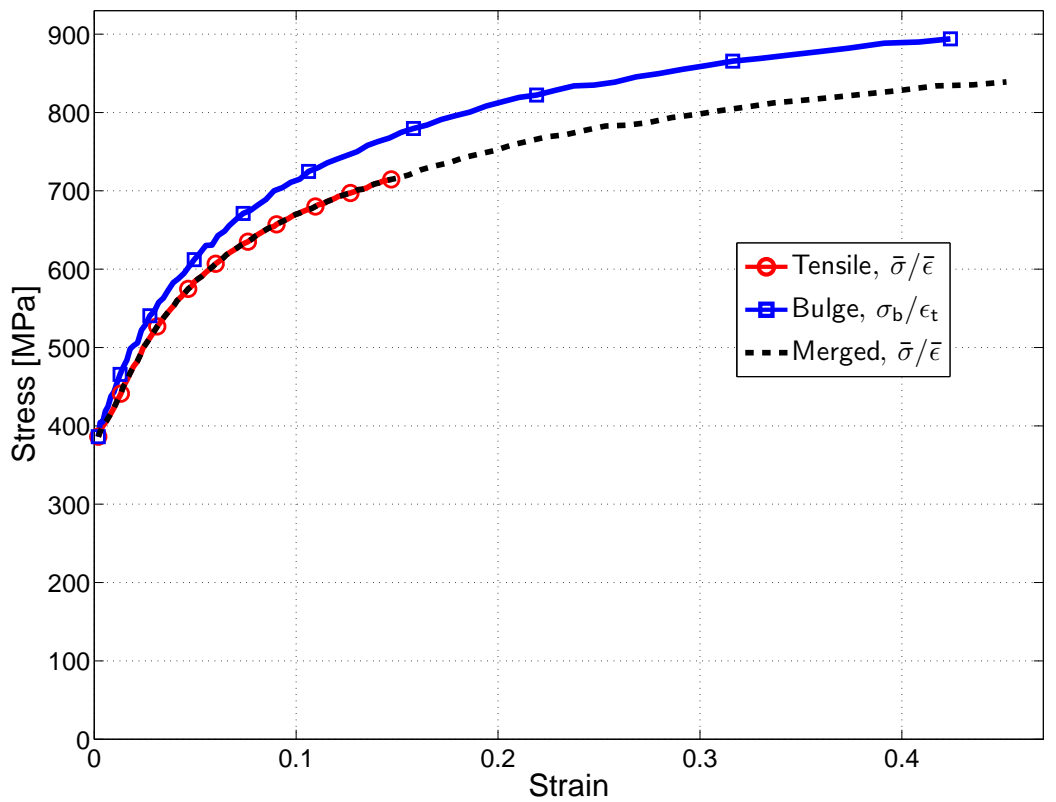

Figure 7: Balanced biaxial stress - thickness strain relation for the bulge test and effective stresseffective strain relations for the uniaxial tensile test and the merged curve.

\section{Simulation methods}

The FE simulation procedure is outlined in Fig. 1. The numerical analyses are performed using the FE code LS-DYNA (see Hallquist (2009)). After each simulation step a file is created that contains essential history data for the next simulation step, i.e., the geometry, stress and strain states of the components.

The simulation procedure starts with a simulation of the forming process, using an explicit FE methodology. It is followed by a springback analysis using an implicit FE methodology. From the net shape of a stamped component, the two ends are cut out to form components 2 and 3. These new components exhibit further springback, which is handled by an additional springback analysis. The simulation procedure then proceeds with the assembly phase. All simulations of this phase are performed using implicit FE methodology with contact interfaces between all parts. The assembly fixture and all other tools are assumed to be rigid. The individual 
components are positioned in the assembly fixture. They are first clamped to the fixture and then their flanges are clamped to each other. During the simulation of the joining process the electrodes forces are applied to rigid electrodes to make sure there is contact between the parts at the locations of the spot welds. Each spot weld is then modelled using four solid elements. Finally a springback analysis is performed on the fully assembled structure in order to predict the final geometry and mechanical properties of the assembled component. In the following sub-sections each simulation step is explained in more details.

\subsection{Forming}

All sheet metal components are modelled using fully integrated quadrilateral shell elements with seven through-thickness integration points. This number of integration points was found from a convergence study. The initial size of a blank element is about $6 \mathrm{~mm}$ and with three levels of adaptation it result in a minimum element size of $1.5 \mathrm{~mm}$ in critical areas. The contacts are defined by a penalty based contact algorithm and Coulomb's law of friction, where the friction coefficient is set to 0.15 . The friction coefficient was chosen after an inverse analysis where the sheet draw-in results from the simulation were compared to experimental data.

\subsection{Springback}

When the die tools are being removed the deformed blank undergoes yet another substantial geometric change due to springback. The amount of springback is governed by the unbalanced residual stress distribution and the elastic stiffness of the material. This fact indicates the importance of a correct description of the stress evolution during stamping, i.e., hardening behaviour, but also the need to have an accurate description of the degradation of elastic stiffness.

Mesh coarsening is applied before the springback analysis to reduce the model size, and thus computational cost and memory requirement. In the chosen case study, components 2 and 3 need to be subjected to two springback simulations. The first one is performed before the trimming operation of the stamped component. Then another springback analysis is performed on each trimmed component such that its new equilibrium state is reached.

\subsection{Positioning}

The parts are positioned so that the holes and slots are aligned with their respective pin on the assembly fixture. It is then followed by a gravity loading analysis, which is performed to ensure a correct placement of the parts on the vertical supports. This analysis step is especially important if large flexible parts are used, since they can deform substantially due to gravity.

\subsection{Clamping}

A number of different techniques can be used to model the clamping operation. The contact surface of the clamps can be modelled or the boundary conditions can be applied on the nodes of the flanges directly. The boundary condition can be either a prescribed load or a prescribed displacement. During this study it was found that the most stable method to apply the clamping was to model the clamps as rigid discs, and using a prescribed displacement. Each part is clamped to the fixture with three clamps, one for each vertical support.

In the experimental set-up, the flanges are clamped together using clamps which have no vertical supports. Thus, the final positions of the clamps are adapted to the deformation of the components. One solution to model this is to apply a relative motion between the clamps, e.g., by shrinking the length of a truss element connecting the clamps. This method retains the benefits 
of a prescribed displacement, such as a controlled deformation, but with less constraint on the deformation path of the model. This means that the flanges will meet in an equilibrium position.

A simplification, made when clamping the components to the fixture, is that all the clamps are moved simultaneously, thus neglecting the clamping sequence. Conversely, when clamping the flanges it is important to take the clamping sequence into account, since the clamping inflicts a large deformation on the components. By controlling the movement of the individual clamps it is possible to simulate different clamping sequences. Thus, further studies can be conducted in order to find an optimal clamping sequence.

\subsection{Welding}

The spot welding process causes the assembly to deform. This is both due to the mechanical force exerted by the welding electrodes as well as by the local thermal expansion and contraction occurring during welding. In this work the thermal effects have been neglected, but the mechanical aspect of the process is modelled; i.e., the electrodes forcing the flanges into contact at each spot weld location. The electrodes are modelled using rigid solid elements. Since only the mechanical aspect of the welding process is modelled, the influence of the welding sequence is estimated to be small. Thus, all electrode forces are applied simultaneously. The resulting spot welds are modelled using four solid elements, which are tied to the nodes of the flanges.

\subsection{Releasing}

At this final assembling stage all clamps are removed simultaneously and a final springback analysis is performed. At this stage it is possible to evaluate if the assembly has reached its specification regarding geometry and/or residual stresses. The resulting state can also be used in further assembling processes and/or in any simulation of the assembly in function.

\section{Results and discussion}

In this section the results from the proposed simulation procedure applied to the test case will be validated against physical test results. Three assemblies have been produced to give some information about the scatter in the physical processes.

As previously mentioned, two sets of CMM measures are performed during the assembling process. First the individual components are measured after clamping to the fixture (Fig. 5a) and secondly the final assembly is measured (Fig. 5b. In Tables 2 and 3 the flange and wall angles at the two ends of the final assembly, and the corresponding sub-component angles, are presented. The angles are defined in accordance with Fig. 8 and they are evaluated using the two extreme experimental measurement positions on each flange/wall. The experimental angles are acquired by using the median value at each measurement position.

The predicted cross-sections of component 1 after clamping to the fixture (see Fig. 9 and Table 2) show an excellent agreement with experimental data. However, it is unfortunate that no experimental data of the unclamped component is available; the clamping to the fixture has eliminated the twist of the component in its length direction.

For components 2 and 3 the agreement of the predictions to experimental data is generally very good, except for the right hand side flanges (see Figs. 10, 11 and Table 2). This disagreement may be caused by deformations due to the manual cutting of the test components.

The predicted cross-sections of the final assembled structure correlate well to the test data (see Fig. 12 and Table 3). One notes that even though the flange angle on the right hand side of 

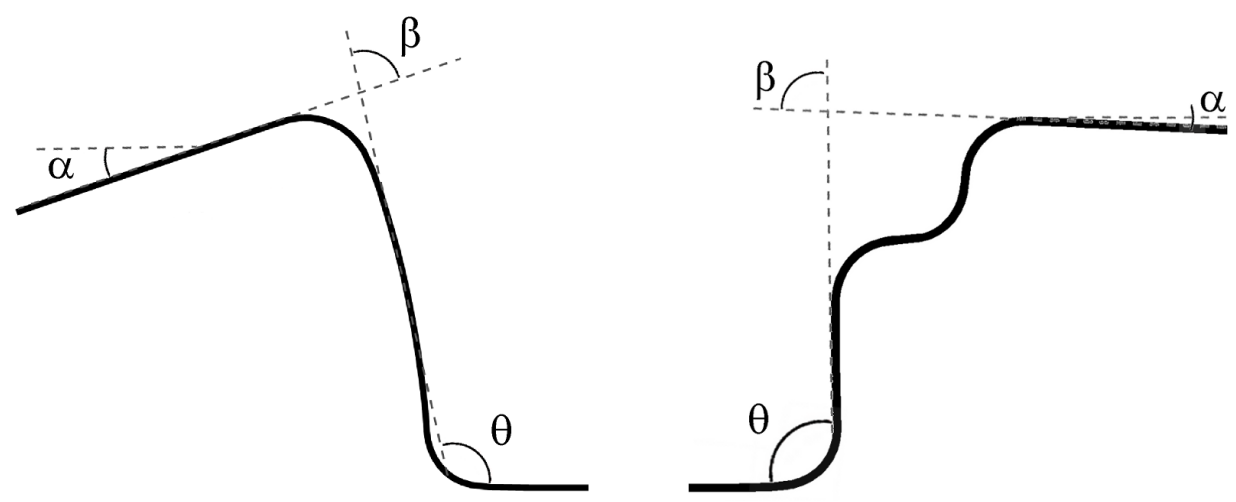

Figure 8: Springback measures, where $\theta$ is the sidewall angle, $\beta$ is the flange angle and $\alpha$ is the deviation from nominal geometry.
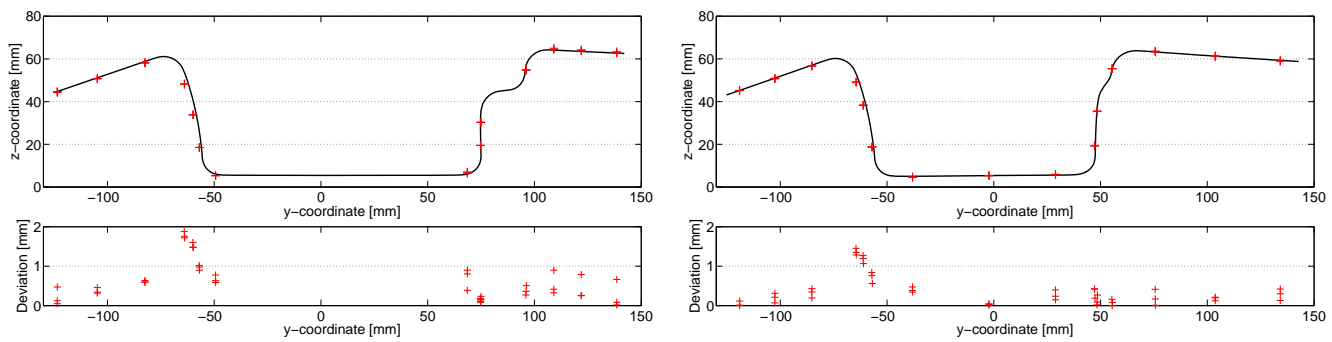

(a) Section 1.1

(b) Section 1.2
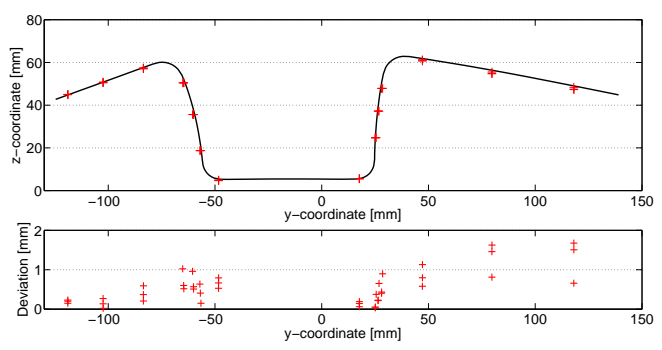

(c) Section 1.3

Figure 9: Cross-sections of component 1. The experimentally measured data are marked by crosses and the black line denotes simulation results. The lower parts of the figures show the absolute deviation between experimental data and simulation results.

component 3 (see Section 3.2 in Table 2) lacks in accuracy, the corresponding flange angle on the assembly, see Section 5 in Table 3, is accurate. This can be explained by the fact that component 1 is stiffer than component 3 , and thus will govern the deformation behaviour.

A major source for errors in the simulation of the assembly may be the clamping. Due to 

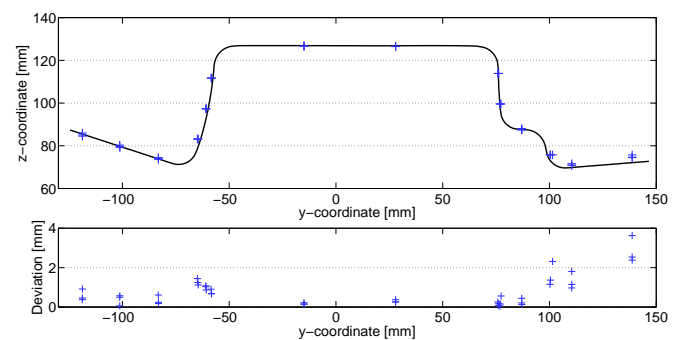

(a) Section 2.1
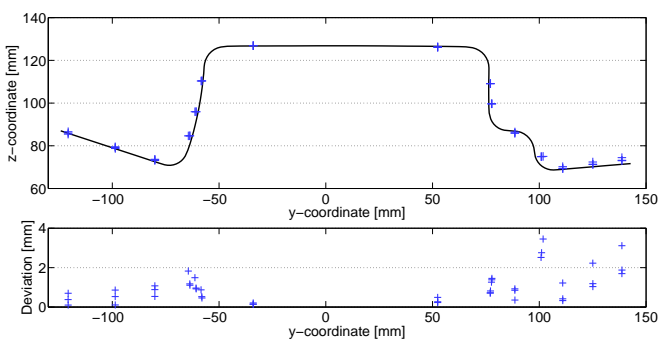

(b) Section 2.2

Figure 10: Cross-sections of component 2. The experimentally measured data are marked by crosses and the black line denotes simulation results. The lower parts of the figures show the absolute deviation between experimental data and simulation results.
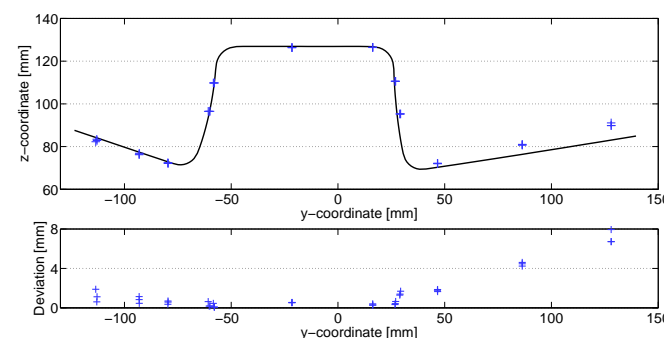

(a) Section 3.1
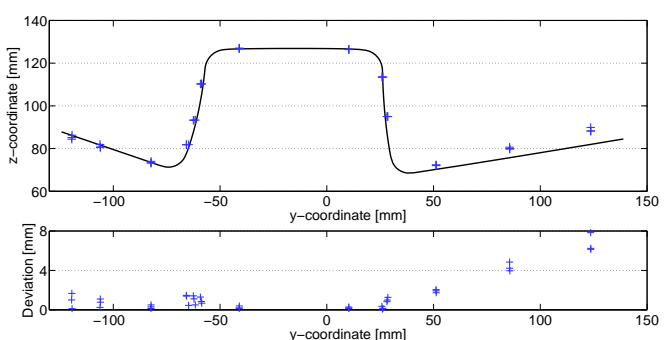

(b) Section 3.2

Figure 11: Cross-sections of component 3. The experimentally measured data are marked by crosses and the black line denotes simulation results. The lower parts of the figures show the absolute deviation between experimental data and simulation results.

the nature of the experimental set-up, where the flanges were clamped manually with handheld clamps without support, there is a problem to exactly reproduce the boundary condition in the simulation model.

The angle of the flange on the right hand side in Figs. 12d and $12 \mathrm{e}$ is in the first case overpredicted and in the latter case underpredicted. Consequently the results from the simulation procedure over predicts the slope of the flange in the length-direction of the structure. This discrepancy could stem from the neglection of heat effects during welding in the simulation model.

There is a tendency, seen in Fig. 12, that the results from the simulations seem to be translated around $1 \mathrm{~mm}$ in the $\mathrm{z}$-direction compared with the experiments. A local discrepancy at the bottom of sub-component 1 , in the area where the assembly rests on the vertical support of the fixture, could be an explanation. Unfortunately no measurements to validate this are available.

Other possible explanations for the small discrepancies seen can be component and assembly process variations. 

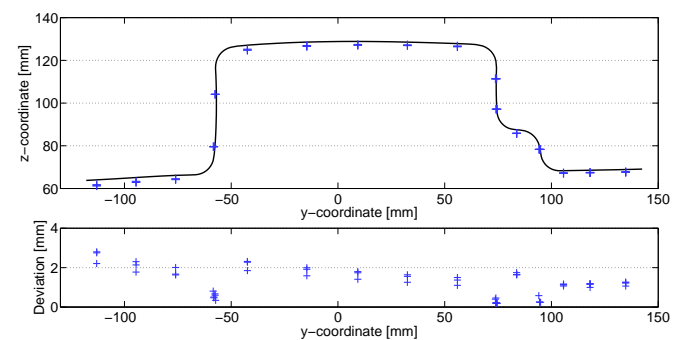

(a) Section 1
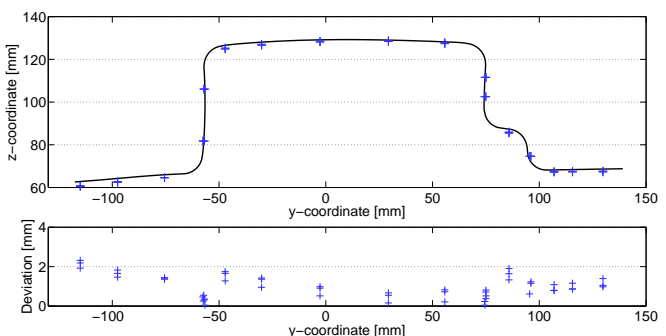

(b) Section 2
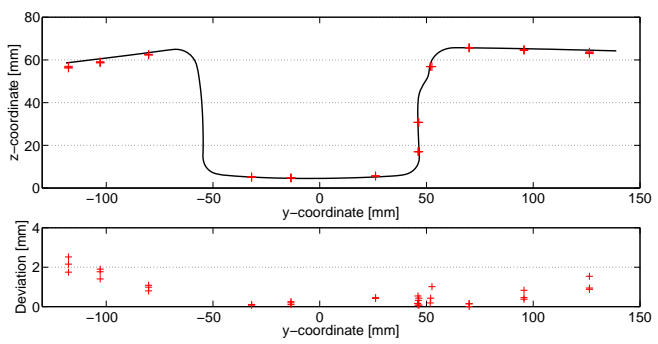

(c) Section 3
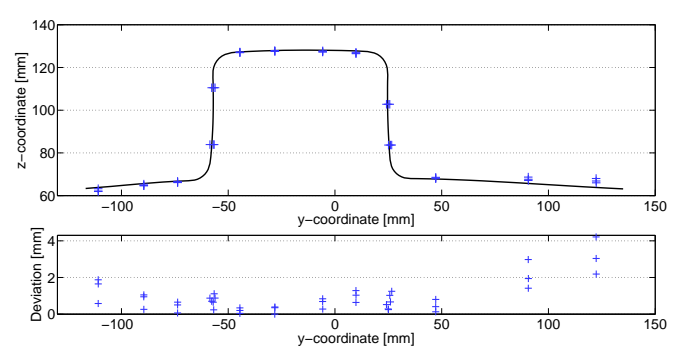

(d) Section 4

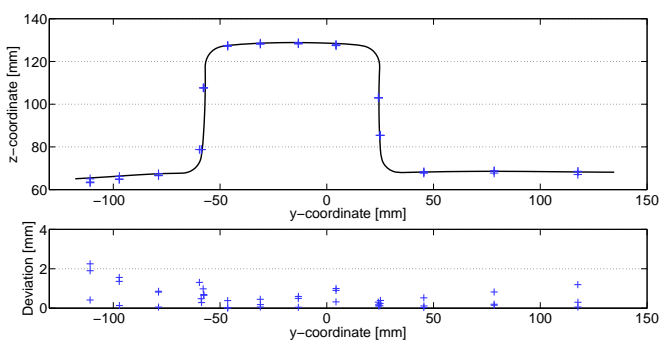

(e) Section 5

Figure 12: Cross-sections of the final assembly. The experimentally measured data are marked by crosses and the black line denotes simulation results. The lower parts of the figures show the absolute deviation between experimental data and simulation results.

\section{Conclusions}

In this work the properties of a sheet metal assembly was to be predicted by acknowledging the contributions from each step of the manufacturing process chain to the properties of the final assembly. This is achieved by using FE simulation methodology to sequentially simulate each step of the manufacturing process chain. The result from each step is transferred to the subsequent step. Thus, the evolution of deformations and residual stresses throughout the manufacturing process chain can be predicted. For validation purpose an assembly was manufactured, both physically and virtually using the proposed simulation procedure. The comparison between predicted and physical test results shows that it is possible to achieve accurate results. Thus, the proposed simulation procedure may be a useful tool to evaluate both the manufacturing processes 
Table 2: Angles of the wall and flange of the components after clamping to the fixture.

\begin{tabular}{ccccccccc}
\hline Section & Flange & & $\theta$ & $\beta$ & $\alpha$ & $\Delta \theta$ & $\Delta \beta$ & $\Delta \alpha$ \\
\hline \multirow{2}{*}{1.1} & \multirow{2}{*}{ Right } & Exp. & 90.4 & 87 & 3.4 & & & \\
& & Sim. & 89.4 & 86.6 & 2.7 & -1.0 & -0.4 & -0.7 \\
\cline { 3 - 9 } & \multirow{2}{*}{ Left } & Exp. & 103 & 84.4 & 18.5 & & & \\
& & Sim. & 101.6 & 82.7 & 18.9 & -1.4 & -1.7 & 0.4 \\
\hline \multirow{2}{*}{1.3} & \multirow{2}{*}{ Right } & Exp. & 97.2 & 86.4 & 10.8 & & & \\
& & Sim. & 96.2 & 86 & 10.2 & -1.0 & -0.4 & -0.6 \\
\cline { 2 - 9 } & \multirow{2}{*}{ Left } & Exp. & 104.2 & 84.8 & 19.3 & & & \\
& & Sim. & 103.5 & 83.5 & 20 & -0.7 & -1.3 & 0.7 \\
\hline \multirow{2}{*}{2.1} & \multirow{2}{*}{ Right } & Exp. & 94.1 & 86.7 & 7.4 & & & \\
& & Sim. & 92.9 & 88.3 & 4.6 & -1.2 & 1.6 & -2.8 \\
\cline { 2 - 9 } & \multirow{2}{*}{ Left } & Exp. & 102.4 & 85.2 & 17.1 & & & \\
& & Sim. & 101.4 & 83.3 & 18.1 & -1.0 & -1.9 & 1.0 \\
\hline \multirow{2}{*}{3.2} & \multirow{2}{*}{ Right } & Exp. & 96.3 & 83.8 & 12.5 & & & \\
& & Sim. & 93.7 & 84.5 & 9.2 & -2.6 & 0.7 & -3.3 \\
\cline { 2 - 8 } & \multirow{2}{*}{ Left } & Exp. & 102.2 & 84.6 & 17.6 & & & \\
& & Sim. & 102.6 & 83.8 & 18.9 & 0.4 & -0.8 & 1.3 \\
\hline
\end{tabular}

Table 3: Angles of the wall and flange for the final assembly.

\begin{tabular}{ccccccccc}
\hline \multirow{2}{*}{ Section } & Flange & & $\theta$ & $\beta$ & $\alpha$ & $\Delta \theta$ & $\Delta \beta$ & $\Delta \alpha$ \\
\hline \multirow{2}{*}{1} & \multirow{2}{*}{ Right } & Exp. & 91.1 & 90.2 & 0.9 & & & \\
& & Sim. & 91.1 & 90.0 & 1.1 & 0 & -0.2 & 0.2 \\
\cline { 2 - 8 } & \multirow{2}{*}{ Left } & Exp. & 91.4 & 96.3 & 4.9 & & & \\
& & Sim. & 91.6 & 94.8 & 3.3 & 0.2 & -1.5 & -1.6 \\
\hline \multirow{2}{*}{5} & \multirow{2}{*}{ Right } & Exp. & 92.4 & 92.3 & 0.1 & & & \\
& & Sim. & 91.0 & 91.0 & 0.0 & -1.4 & -1.3 & -0.1 \\
& \multirow{2}{*}{ Left } & Exp. & 92.4 & 97.8 & 5.4 & & & \\
& & Sim. & 92.8 & 96.2 & 3.5 & 0.4 & -1.7 & -1.9 \\
\hline
\end{tabular}

and the final assembly. However, further studies are needed to analyse the effects of the clamping and spot welding sequences and to optimise the manufacturing process parameters. Furthermore, variation is an inevitable part of the production process. Material and process variations can drastically change the results. Consequently, further studies are needed in order to foresee the effect of variations in the involved processes. This would be of a great value when choosing the correct process parameters and tolerance settings of the manufacturing process of the product and its constituent sub-components.

\section{Acknowledgements}

The material characterisation and stamping of the components were conducted by Per Thilderkvist and Jörgen Hertzman at the Industrial Development Center, Olofström, Sweden. The physical assembling and CMM measurements were carried out at Volvo Car Body Components, Olofström, Sweden, and the support from Alf Andersson is greatly acknowledged. The work presented in 
this paper has been carried out with financial support from the Swedish foundation for strategic research, the ProViking programme.

Ahmetoglu, M., Kinzel, G., Altan, T., 1994. Computer simulation for tool and process design in sheet forming. Journal of Materials Processing Technology 46, 421-441.

Andersson, A., 2007. Numerical and experimental evaluation of springback in advanced high strength steel. Journal of Materials Engineering and Performance 16, 301-307.

Banabic, D., 2000. Formability of Metallic Materials. Springer Verlag, Berlin.

Barlat, F., Brem, J.C., Yoon, J.W., Chung, K., Dick, R.E., Lege, D.J., Pourboghrat, F., Choi, S.H., Chu, E., 2003. Plane stress yield function for aluminum alloy sheets - part 1: Theory. International Journal of Plasticity 19, $1297-1319$.

Barlat, F., Lian, K., 1989. Plastic behavior and stretchability of sheet metals. Part I: A yield function for orthotropic sheets under plane stress conditions. International Journal of Plasticity 5, 51 - 66.

Cai, W., Hsieh, C., Long, Y., Marin, S., Oh, K., 2006. Digital panel assembly methodologies and applications for compliant sheet components. Journal of Manufacturing Science and Engineering. Transactions of the ASME 128, 270-279.

Eggertsen, P.A., Mattiasson, K., 2009. On the modelling of the bending-unbending behaviour for accurate springback predictions. International Journal of Mechanical Sciences 51, 547 - 563.

Eggertsen, P.A., Mattiasson, K., 2010. On constitutive modeling for springback analysis. International Journal of Mechanical Sciences 52, $804-818$

Galbraith, C., Thomas, D., Finn, M., 2003. Manufacturing simulation of an automotive hood assembly. 4th European LS-DYNA Users Conference Metal Forming III, E-III - 37-44. Ulm.

Hallquist, J., 2009. LS-DYNA Theory Manual. Livermore Software Technology Corporation. Livermore.

Hammett, P.C., Majeske, K.D., Baron, J.S., 1998. Functional build: Integrating automotive body-design \& processdevelopment, pp. 321-327.

Hill, R., 1948. A theory of the yielding and plastic flow of anisotropic metals. Proceedings of the Royal Society of London. Series A, Mathematical and Physical Sciences 193, pp. 281-297.

Lee, S., Yang, D., 1998. An assessment of numerical parameters influencing springback in explicit finite element analysis of sheet metal forming process. Journal of Materials Processing Technology 80-81, 60-67.

Li, K., Carden, W., Wagoner, R., 2002. Simulation of springback. International Journal of Mechanical Sciences 44, $103-122$.

Liu, S.C., Hu, S.J., 1997. Variation simulation for deformable sheet metal assemblies using finite element methods. Journal of Manufacturing Science and Engineering, Transactions of the ASME 119, 368-373.

Makinouchi, A., 1996. Sheet metal forming simulation in industry. Journal of Materials Processing Technology 60, $19-26$.

Makinouchi, A., Teodosiu, C., Nakagawa, T., 1998. Advance in FEM simulation and its related technologies in sheet metal forming. CIRP Annals - Manufacturing Technology 47, $641-649$.

Morestin, F., Boivin, M., 1996. On the necessity of taking into account the variation in the Young modulus with plastic strain in elastic-plastic software. Nuclear Engineering and Design 162, 107 - 116.

Papadakis, L., 2008. Simulation of the Structural Effects of Welded Frame Assemblies in Manufacturing Process Chains. Ph.D. thesis. Technische Universität München. München

Papeleux, L., Ponthot, J.P., 2002. Finite element simulation of springback in sheet metal forming. Journal of Materials Processing Technology 125-126, 785-791.

Sigvant, M., Mattiasson, K., Vegter, H., Thilderkvist, P., 2009. A viscous pressure bulge test for the determination of a plastic hardening curve and equibiaxial material data. International Journal of Material Forming , 1-8.

Skowronski, V.J., Turner, J.U., 1997. Using Monte-Carlo variance reduction in statistical tolerance synthesis. ComputerAided Design 29, 63 - 69.

Wagoner, R., Li, M., 2007. Simulation of springback: Through-thickness integration. International Journal of Plasticity $23,345-360$.

Yoshida, F., Uemori, T., 2003. A model of large-strain cyclic plasticity and its application to springback simulation. International Journal of Mechanical Sciences 45, 1687 - 1702.

Yoshida, F., Uemori, T., Fujiwara, K., 2002. Elastic-plastic behavior of steel sheets under in-plane cyclic tensioncompression at large strain. International Journal of Plasticity 18, $633-659$.

Zhang, L., Zhu, X., Xu, S., Wang, C.T., 2009. Advanced simulation technology using LS-DYNA for automotive body manufacturing process: From stamping to assembly. SAE World Congress \& Exhibition Sheet/ Hydro/ Gas Forming Technology and Modeling (Part 1 of 3). Detroit. 